\title{
¿En qué lenguas se hace ciencia? La gestión del multilingüismo en el Parc Científic de Barcelona ${ }^{1}$
}

\author{
F. Xavier Vila \\ Universitat de Barcelona, CUSC \\ fxvila@ub.edu \\ Vanessa Bretxa \\ Universitat de Barcelona, CUSC \\ vanessa.bretxa@ub.edu \\ Llorenç Comajoan \\ Universitat de Vic, CUSC \\ lcomajoa@gmail.com
}

Resumen: Este estudio investiga los usos lingüisticos en catalán, castellano e inglés de los investigadores del Parc Cientific de Barcelona, un entorno con características sociolingüísticas heterogéneas. Los resultados del análisis de conglomerados muestran que el personal investigador ( $n=179$ ) se estructura alrededor de cuatro grupos según su competencia y usos lingüisticos y proporcionan evidencia de que a) en instituciones cientificas académicas en entornos multilingües los procesos de cambio lingüistico no se producen de manera compacta $y$ b) de que el inglés no se convierte necesariamente en la lengua franca de la institución.

Palabras clave: lengua, plurilingüismo, globalización, investigación, usos lingüisticos, universidad.

1 El estudio que se presenta se inició a raíz de un encargo del entonces Vicerrectorado de Política Lingüística de la Universidad de Barcelona (UB) al Centro Universitario de Sociolingüística y Comunicación (CUSC-UB), el cual desembocó en el informe Vila, Comajoan, Bretxa y Arroyo (2010). Este trabajo ha contado con la colaboración del Grupo de Estudio de la Variación (2009 SGR 521) de la AGAUR de la Generalitat de Catalunya. 
Which Languages are used in Science?

Managing Multilingualism at the Barcelona Science Park

Abstract: This study investigates the use of Catalan, Spanish, and English by researchers in the sociolinguistically heterogeneous environment of the Parc Cientific de Barcelona (Barcelona Science Park). Cluster analysis results show that researchers $(n=179)$ can be divided into four groups according to their language competence and usage and also provide evidence that a) in multilingual scientific academic institutions language change processes do not occur in a compact manner and b) that English does not necessarily become the lingua franca of the institution.

Keywords: language, multilingualism, globalization, research, language use, university. 


\section{Lenguas e investigación en un entorno globalizado}

\subsection{La globalización y el multilingüismo}

La sociología de la lengua se incorporó con cierto retraso al análisis de la globalización y sus efectos (Coupland, 2010). Sin embargo, desde hace una década, la disciplina avanza en el estudio de las consecuencias de este proceso sobre lo que Albert Bastardas (2007) ha denominado la linguosfera, es decir, el conjunto de ecosistemas lingüísticos del planeta.

Como en el resto de ciencias sociales, la conceptualización de lo que la mundialización implica dista mucho de ser homogénea. Autores como Fairclough (2006) y Ricento (2010: 123) han propuesto enmarcar las opiniones existentes sirviéndose de la clasificación de Held et alii (1999) en biperglobalistas, escépticos y transformacionalistas. Pero el intento de encuadrar la investigación sociolingüística en este marco no acaba de resultar satisfactorio porque esta clasificación mezcla lo analítico con lo valorativo. En este sentido, a la hora de entender los estudios sociolingüísticos sobre la globalización hay que distinguir claramente entre tres variables.

La primera variable que distingue estos estudios es su posición en relación con la novedad del fenómeno. Para algunos investigadores, las transformaciones generadas por la globalización en la linguosfera son de gran envergadura, hasta el punto de que hablan de cambios sin precedentes en la historia de la humanidad. Son autores que plantean la aparición de un nuevo orden lingüístico internacional (Fishman, 1999) o la creación de un sistema global de lenguas (Calvet, 1999; de Swaan, 2001, 2010). Por otro lado, un número menor pero significativo de autores argumenta que los cambios ecolingüísticos emparejados con la globalización son substancialmente comparables con otros cambios acaecidos en el pasado e incluso tienen menor trascendencia que aquellos. En esta línea destaca especialmente la reflexión de Mufwene (2010), quien arguye que la indoeuropeización de Europa, la bantuización de África y la europeización de América y Oceanía tuvieron más calado que la simple expansión contemporánea del inglés como lengua franca. Entre ambos polos, autores como Coupland (2010) argumentan que la globalización es un fenómeno complejo con algunas dimensiones nuevas aunque enraizado en tendencias de largo alcance histórico.

La segunda gran variable tiene que ver con la dirección y el alcance de los cambios. Nadie duda de que se esté produciendo una reducción veloz y sin precedentes en el número de lenguas en el mundo. También está claro que el inglés no solo se ha afianzado en los territorios históricamente anglófonos y en sus antiguas colonias, sino que además se está extendiendo como lengua franca más allá de es- 
tos países (Kachru, Kachru y Nelson, 2006) y es mundialmente hegemónico en ámbitos como la ciencia (Ammon, 2010). Pero lo que no está tan claro es si esta expansión va a seguir en un futuro y, en caso de que sea así, tampoco está claro que su centro de gravedad vaya a continuar hallándose en los países anglófonos, o si se ubicará fuera de ellos como lengua verdaderamente internacional (Graddol, 2006; Ostler, 2010). Tampoco queda claro el verdadero alcance de la estampida hacia el inglés (de Swaan, 2001). Para un primer grupo de investigadores, como Phillipson (1992, 2003) y Nettle y Romaine (2000), el inglés _la lengua asesina - sería el principal responsable de la reducción acelerada de la diversidad lingüística mundial. Por el contrario, un nutrido grupo de autores (Fishman, 1999; Mufwene 2008, 2010) advierten de que la extinción lingüística está siendo protagonizada principalmente por las lenguas de los respectivos estados nación, que son las que arrinconan hasta la extinción las lenguas sin estado propio ni propicio.

La tercera variable para clasificar los estudios sobre la globalización lingüística es cómo valoran la agencia de los cambios lingüísticos. Un contingente significativo de autores, como Calvet y Varela (2000), Blommaert (2010), Mufwene (2008, 2010) u Ostler (2010), considera que los cambios son esencialmente una consecuencia de relaciones humanas en términos de poder político y económico, y pone en segundo lugar las motivaciones glotopolíticas. En cambio, otros autores, como Phillipson (1992, 2003, 2006) y Skutnabb-Kangas y Phillipson (2010), destacan que existe una voluntad política explícita cuando menos por parte de algunos agentes políticos de liquidar la diversidad lingüística, hasta el punto de denunciar como lingüicidas buena parte de las políticas lingüísticas vigentes.

Desde un punto de vista analítico, resulta también importante distinguir a los autores que adoptan como tema de estudio la lengua de los que se centran en el repertorio. Mientras que los primeros tienden a analizar el mantenimiento y la substitución lingüística de variedades idiomáticas, los segundos suelen concentrar su interés en cómo la globalización afecta a los recursos lingüísticos de individuos o comunidades. Así, es frecuente que los primeros se preocupen por la extinción de las lenguas minoritarias, mientras que los segundos tienden a remarcar que las lenguas expansivas se indigenizan y pasan a formar parte de los ecosistemas locales (Blommaert, 2010; Coupland, 2010).

Finalmente, es importante distinguir el componente valorativo de la investigación en este campo. En este sentido, hay que señalar que, independientemente de la posición de cada autor, en el campo de la sociolingüística apenas se detecta el entusiasmo por la unificación emanada de la globalización que sí se percibe claramente en ciertos autores económicos o politólogos. Bien al contrario, buena par- 
te de la bibliografía sobre el tema contempla con consternación la desaparición cotidiana del patrimonio lingüístico universal y, si bien no son pocos los autores que saludan la aparición de una hipotética lengua franca mundial, suelen hacerlo enfatizando que esta unificación debería realizarse manteniendo la diversidad prexistente, y no suprimiéndola (Bastardas, 2007).

\subsection{Las lenguas en ámbitos científicos y académicos}

Una de las esferas más afectadas lingüísticamente por la globalización es el campo de la ciencia y la universidad (Ammon, 2012; Vila, 2012; Vila y Bretxa, en preparación). Por su propia configuración histórica, su intrínseca apertura al exterior y su dependencia de instituciones internacionales, ambos campos sociales aparecen abiertos a la irrupción de las lenguas francas. Desgraciadamente, los estudios sobre esta área apenas han empezado a aparecer y los trabajos disponibles sobre la ecología lingüística en instituciones de investigación sugieren de entrada que hay que diferenciar claramente entre las prácticas lingüísticas más estrictamente investigadoras, encarnadas en las publicaciones y los congresos internacionales, y el resto de prácticas lingüísticas académicas y universitarias.

En lo que atañe al primero de estos ámbitos, la situación parece relativamente sencilla de comprender. Abandonado el latín como lengua franca académica a lo largo del siglo xvinI (Baggioni, 1997; Aracil, 2004), diferentes lenguas estatales europeas - francés, alemán, ruso, etc. - mantuvieron una pugna por reemplazarlo. Sin embargo, desde mediados del siglo xx, el inglés fue convirtiéndose en la principal lengua de las publicaciones científicas, en un proceso que ha reducido la presencia de las otras lenguas y que no muestra signos de revertir (Ammon, 1999, 2010; Ostler, 2010: 207).

Ahora bien, fuera de los ámbitos más genuinamente globalizados, la ecología lingüística académica y universitaria parece ser mucho menos inclemente para la diversidad lingüística. El abandono del latín no desembocó en la adopción de un nuevo vehículo lingüístico compartido para desempeñar las funciones académicas. Bien al contrario, ya que a medida que se expandía el sistema universitario tanto por Occidente como por amplísimas áreas de Asia, los centros de enseñanza e investigación se fueron abriendo progresivamente a docenas de lenguas nacionales, incluyendo no solo a las de los estados nación, sino también a las de la nacionalidades sin estado (Arzoz, 2012; Vila y Bretxa, en preparación). Y siguiendo la demanda generada por las crecientes necesidades docentes multilingües, también se desarrollaron un número considerable de recursos universitarios en estas lenguas nacionales. En este mismo sentido, y a pesar de la creciente internacionalización de la investigación, son aparentemente escasos los centros 
universitarios no ubicados en territorios anglófonos o poscoloniales cuya gestión y administración se haya transferido desde las lenguas nacionales hacia el inglés, con la excepción de los centros realmente internacionales. En pocas palabras, a pesar de la extensión del inglés como lengua franca académica y científica, todo parece sugerir que las lenguas nacionales autóctonas son ampliamente mayoritarias en la docencia en todos los países de Europa y buena parte de Asia.

\section{El proyecto «Los conocimientos, los usos y las necesidades lingüísticos del $\mathrm{PCB}$ »}

Es en el contexto de la investigación sobre la gestión del multilingüismo en ámbitos universitarios y académicos donde debe ubicarse el estudio aquí presentado sobre el Parc Científic de Barcelona (PCB). Los parques científicos son instituciones de naturaleza pública o privada, establecidas a menudo en el seno de universidades, que albergan equipos de investigación. Estos parques están siendo promovidos para aprovechar recursos y crear sinergias, conseguir economías de escala y facilitar la transferencia de conocimiento. En este sentido, los parques no solo se dirigen a la investigación básica, sino que favorecen la constitución de iniciativas mixtas entre universidad y empresa privada.

El PCB fue creado en 1997 en la Universidad de Barcelona y fue el primer parque científico del Estado español. Sus objetivos eran potenciar la investigación de excelencia, dinamizar la relación entre la universidad y la empresa, impulsar la creación de nuevas empresas e institutos y promover el diálogo ciencia-sociedad y las vocaciones científicas. En el momento de la investigación, se ubicaban en el PCB cuatro institutos de investigación, más de cincuenta empresas, una incubadora de empresas biotecnológicas y más de setenta grupos de investigación.

El PCB tiene una vocación de internacionalización y en buena parte de sus dependencias trabajan personas de procedencia y origen lingüístico diversos. Desde el punto de vista idiomático, el PCB constituye una institución social de gran complejidad, cuyos miembros combinan una considerable diversidad sociocultural de partida con una necesidad imperiosa de establecer rutinas de funcionamiento que les permitan el trabajo cooperativo. Así pues, igual que el resto de instituciones del sistema universitario y de investigación de Cataluña, el centro constituye un entorno especialmente interesante para el estudio de la ecología lingüística por cuanto coinciden en él tres tendencias sociolingüísticas contradictorias que podemos describir en términos de atracciones contrapuestas.

En primer lugar, en cuanto que institución ubicada en Cataluña, creada por una universidad pública catalana, el PCB siente la atracción lógica hacia el uso de 
la lengua catalana, lengua propia y oficial de la institución y el país. En segundo lugar, en cuanto que centro dependiente en último término de la Administración española, el centro se ve atraído hacia el uso de la lengua castellana, lengua oficial del Estado y oficial también en Cataluña. Finalmente, en cuanto que centro de investigación con proyección internacional, el PCB se siente atraído hacia el uso del inglés.

El estudio aquí presentado se propuso como objetivo describir los repertorios lingüísticos (Gal, 1987; Blommaert, 2010) de los integrantes del PCB y contrastarlos con las normas de elección lingüística que regulaban la convivencia entre individuos, grupos de trabajo y la administración del PCB. Además, se pretendía entender cuáles eran las opiniones de este colectivo humano sobre la gestión lingüística de la institución y explorar sus necesidades idiomáticas. El estudio partía de una perspectiva sociolingüística crítica según la cual, en la vida real, el acceso a los diferentes elementos del repertorio lingüístico local no es igualitario, y que en cada campo social y cada mercado (local, nacional, estatal o internacional) se imponen unas valoraciones de los distintos capitales lingüísticos en juego (Bourdieu, 1982; Boix y Vila, 1998; Heller, 2002). Estas valoraciones tienen repercusiones en el comportamiento de los individuos: determinan los esfuerzos que realizan para alcanzar determinados conocimientos, dibujan las prácticas lingüísticas y llegan a modificar las características estructurales de los códigos presentes (Vila, 2012).

Al mismo tiempo, se entendía que la creación de colectivos con necesidades cooperativas como la aquí estudiada conlleva a la creación de comunidades de prácticas (Eckert y McConnell-Ginet, 1999) en que sus integrantes deben sincronizar sus comportamientos comunicativos en torno a unas prácticas compartidas. En términos lingüísticos, esta sincronización implica una tendencia hacia la convergencia lingüística, que podría favorecer la adopción de lenguas francas y una especialización de funciones para las lenguas. En este sentido, en cuanto que colectivo integrado por individuos de orígenes y formaciones diferentes $y$ estructurados en comunidades de prácticas débilmente interrelacionadas bajo un marco administrativo común, el PCB permitía un grado de autonomía y de variación interna considerable en las dinámicas de los equipos de trabajo, de modo que no era descartable que las distribuciones de lenguas de los equipos de trabajo fueran divergentes. En pocas palabras, el análisis de la ecología lingüística del PCB exigía tener muy presente cómo se estructuraba la institución en general y cuáles eran las tareas que realizaban sus colectivos, para permitir que las diferencias entre campos e, incluso, entre sectores e instituciones se hicieran visibles. 
En trabajos anteriores (Vila, Comajoan y Bretxa, 2010, 2012) se ha procedido al análisis global de las prácticas lingüísticas de los integrantes del PCB, mientras que el presente trabajo se centra en los perfiles lingüísticos de los investigadores del PCB.

\section{Metodología}

Para la recogida de datos (realizada durante el curso 2008-2009), se emplearon tres herramientas sociolingüísticas: la encuesta en línea, el análisis del paisaje lingüístico y las entrevistas en profundidad (Vila, Comajoan y Bretxa, 2012). En el presente artículo se presentan los datos de la encuesta sociolingüística (en línea), que se apoyan con algunos datos de las entrevistas personales.

El cuestionario sociolingüístico se estructuró en tres secciones principales, que proporcionaron información sociolingüística particularmente centrada en la competencia y los usos del personal del PCB (Tabla 1).

Tabla 1. Secciones de la encuesta sociolingüística

\begin{tabular}{|l|l|}
\hline \multicolumn{1}{|c|}{ Secciones } & \multicolumn{1}{c|}{ Variables principales } \\
\hline Presentación & $\begin{array}{l}\text { Objetivos de la encuesta y lengua para realizarla (catalán, } \\
\text { castellano o inglés) }\end{array}$ \\
\hline Información general & $\begin{array}{l}\text { Sexo, edad, lugar de nacimiento, tiempo que hace que trabaja en } \\
\text { el PCB, etc. }\end{array}$ \\
\hline $\begin{array}{l}\text { Información } \\
\text { sociolingüística }\end{array}$ & $\begin{array}{l}\text { Lengua inicial, lengua inicial de los padres, lengua usada con los } \\
\text { hijos, etc. }\end{array}$ \\
\cline { 2 - 3 } & $\begin{array}{l}\text { Competencia lingüística en catalán, castellano, inglés y otras } \\
\text { lenguas (del 0 «nada» al 10 «perfectamente»). }\end{array}$ \\
\cline { 2 - 3 } & Usos lingüísticos: internos y externos (orales y escritos) \\
\hline Necesidades & Necesidades de aprendizaje o mejora en lenguas \\
\hline
\end{tabular}

Para el diseño de los ítems de la encuesta sobre competencia y usos se tuvieron en cuenta encuestas recientes de características similares, especialmente la «Estadística de usos lingüísticos en Cataluña 2003» (Torres et alii, 2005). Siguiendo las recomendaciones de Romaní (2005: 135), se pidieron proporciones de uso (del 0 al 100), lo que permite hacer comparaciones con otros estudios que también han usado el mismo tipo de pregunta, como la «Encuesta de usos lingüísticos en las instituciones públicas catalanas» (Renau, 2004). La recogida de datos se realizó mediante una encuesta sociolingüística distribuida en línea con la plataforma Questionpro (www.questionpro.com).

El principal obstáculo para la aplicación del cuestionario fue la identificación del número total de integrantes del PCB debido a la autonomía de cada centro 
del PCB y a la movilidad considerable de su personal. Gracias a la dirección del PCB, se pudo identificar a 700 trabajadores y/o colaboradores y acceder a ellos por correo electrónico. La dirección del PCB envió una invitación para colaborar con la investigación siguiendo un enlace de internet que llevaba directamente a la encuesta. Recibieron la invitación aproximadamente los 700 miembros identificados, repartidos entre personal de investigación y de administración. Un total de 605 miembros llegaron a consultar el enlace y 398 contestaron la encuesta, en parte o de forma completa. En otras palabras, respondió a la encuesta más de la mitad de los contactados $(56,9 \%)$ y dos tercios $(65,8 \%)$ de ellos llegaron a consultar el enlace, unos resultados satisfactorios en encuestas de estas características. Un 58,5\% de los participantes en la encuesta contestaron en catalán, un $31,7 \%$ en castellano y un 9,8\% en inglés. Según la distribución de las respuestas por categorías profesionales, el $59,8 \%$ eran investigadores ( $n=238$ ), el $34,2 \%$ era personal de administración y gestión $(n=136)$ y un $6 \%$ no se identificó $(n=24)$. De los 238 miembros del personal de investigación del PCB que respondieron la encuesta —en parte o de forma completa-, 179 (75,2\%) respondieron todas las preguntas relacionadas con los usos lingüísticos, por tanto, este es el subconjunto de encuestados que forman parte del análisis de conglomerados (cluster analysis) que se presenta en la sección de resultados. ${ }^{2}$

Para analizar la competencia y usos lingüísticos de los investigadores del PCB se utilizó la técnica de reducción de datos, en concreto, la de conglomerados jerárquicos siguiendo el método de Ward ${ }^{3}$ a partir de las variables de usos lingüísticos. El análisis de conglomerados jerárquicos intenta identificar grupos relativamente homogéneos de casos basándose en las características seleccionadas. Para llevarlo a cabo se tuvieron en cuenta 12 indicadores obtenidos de los ítems de la encuesta sobre los usos lingüísticos de los investigadores y se agruparon en dos variables:

a) Comunicaciones internas en el PCB: Qué lengua utiliza cuando... 1) habla con sus compañeros de su grupo de investigación; 2) habla con sus jefes (investigador principal, director, etc.); 3) habla con el personal de gestión y administración; 4) escribe notas informales a sus compañeros de trabajo (en papel, por correo electrónico, etc.), y 5) escribe documentos de gestión (formularios, correspondencia, solicitudes, etc.).

b) Comunicaciones externas en el PCB: Qué lengua utiliza cuando... 1) presenta comunicaciones en congresos de ámbito internacional; 2) presenta comuni-

2 Una explotación de los datos globales, aunque inicial, puede consultarse en Vila, Comajoan y Bretxa (2010, 2012).

3 El método de Ward es uno de los más utilizados para la reducción de datos, tiene casi todas las ventajas del método de la media y suele ser más discriminativo en la determinación de los niveles de agrupación (CEACES, 2000). 
caciones en congresos de ámbito español o latinoamericano; 3) presenta comunicaciones en congresos de ámbito catalán; 4) habla con otros investigadores fuera de su grupo de investigación; 5) escribe trabajos o artículos de investigación; 6) lee libros relacionados con su disciplina de investigación, y 7) lee revistas relacionadas con su disciplina de investigación.

\section{Resultados}

El análisis de conglomerados a partir de las respuestas del personal de investigación $(n=179)$ muestra las distintas agrupaciones de los integrantes del PCB en cuanto a los usos lingüísticos que realizan (Tabla 2).

\section{Tabla 2. Representación jerárquica de los conglomerados del personal de investigación $(n=179)$}

\begin{tabular}{|c|c|c|c|c|c|c|}
\hline $\begin{array}{l}\text { Número de } \\
\text { conglomerados }\end{array}$ & \multicolumn{6}{|c|}{ Conglomerados y número de integrantes } \\
\hline 1 & \multicolumn{6}{|c|}{ C0 (179) } \\
\hline 2 & \multicolumn{3}{|c|}{ C1 (95) } & \multicolumn{3}{|c|}{ C2 (84) } \\
\hline 3 & \multicolumn{3}{|c|}{ C1 (95) } & \multicolumn{2}{|c|}{ C2 (84) } & C3 (14) \\
\hline 4 & C1 (13) & \multicolumn{2}{|c|}{ C2 (82) } & \multicolumn{2}{|c|}{ C3 (70) } & C4 (14) \\
\hline 5 & C1 (13) & \multicolumn{2}{|c|}{$\mathrm{C} 2(82)$} & C5 (16) & C3 (54) & C4 (14) \\
\hline 6 & $\mathrm{C} 1(13)$ & $\mathrm{C} 2(47)$ & C6 (35) & C5 (16) & C3 (54) & C4 (14) \\
\hline
\end{tabular}

Cada una de las segmentaciones según el número de conglomerados permite hacerse una idea, cada vez más precisa, de cómo son sociolingüísticamente los investigadores del PCB. La primera segmentación divide al personal de investigación en dos grandes colectivos: el conglomerado 1 está formado por 95 investigadores, prácticamente todos nacidos en algún territorio de lengua catalana, mientras que el conglomerado 2 está integrado por 84 investigadores de origen variado. La diferencia entre ambos conglomerados es que los primeros hacen un uso muy importante del catalán, mientras que los segundos usan mucho menos este idioma.

Tal como puede apreciarse en la Tabla 2, esta primera división se mantiene en la siguiente segmentación. Si en lugar de segmentar en dos se opta por una segmentación en tres conglomerados, el primero se mantiene incólume, mientras que el segundo se divide en dos: un primer subgrupo está integrado por 70 investigadores españoles, europeos e hispanoamericanos, mientras que el otro subgrupo, más pequeño, está formado por 14 investigadores principalmente europeos 
(no españoles). El rasgo que distingue mayormente a ambos conglomerados es que el primero de estos subgrupos alterna entre el inglés y el castellano, mientras que el segundo se halla instalado en el uso predominante del inglés. El análisis prosiguió con una segmentación en cuatro, cinco y hasta seis conglomerados. En aras de la simplicidad y para evitar la disgregación en grupos excesivamente pequeños, se tomó la decisión de analizar con detenimiento la clasificación en cuatro conglomerados.

\subsection{Los cuatro grandes grupos de investigadores}

El análisis en cuatro conglomerados del personal de investigación del PCB visualiza cuatro grupos o perfiles lingüísticos según el uso que hacen de las distintas lenguas (catalán, castellano e inglés) en su labor profesional (Figura 1). A grandes rasgos, se distingue un primer grupo de investigadores autóctonos, con dominio de catalán y castellano, pero con nivel bajo de inglés (7,3\%). El segundo conglomerado, y el más numeroso, es el formado por investigadores capaces de expresarse en catalán, castellano e inglés (45,8\%). El tercer colectivo engloba a investigadores bilingües en castellano e inglés, pero con escaso dominio del catalán $(39,1)$. Finalmente, el cuarto colectivo es el formado por investigadores con alto nivel de inglés y escaso dominio de catalán y castellano (7,8\%).

\section{Figura 1. Perfiles lingüísticos del personal de investigación (porcentajes) $(\mathbf{n}=179)$}

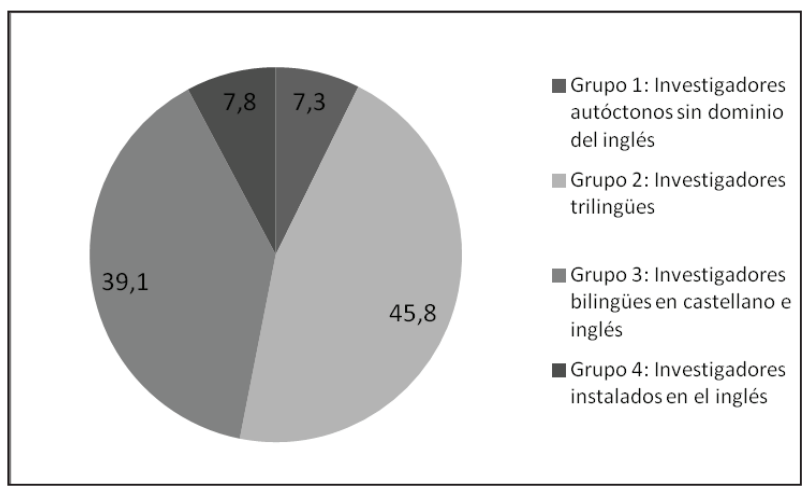

En los apartados siguientes se presentan los principales rasgos sociolingüísticos de cada uno de estos conglomerados, así como su nivel de conocimiento de lenguas. Las características sociales y la competencia lingüística de cada grupo están resumidas en la Tabla 3 (la gradación a mayor gris indica un mayor porcentaje). 
Tabla 3. Características sociales y conocimientos lingüísticos de los cuatro conglomerados

\begin{tabular}{|c|c|c|c|c|c|c|}
\hline Variable & Categorías & $\begin{array}{c}\text { Significación } \\
\left(\chi^{2}\right)\end{array}$ & $\mathrm{C} 1$ & $\mathrm{C} 2$ & $\mathrm{C} 3$ & $\mathrm{C} 4$ \\
\hline$\%$ & & & 7,3 & 45,8 & 39,1 & 7,8 \\
\hline $\mathrm{n}$ & & & 13 & 82 & 70 & 14 \\
\hline \multirow[t]{2}{*}{ Sexo } & Hombre & \multirow{2}{*}{ No } & 61,5 & 51,2 & 54,3 & 57,1 \\
\hline & Mujer & & 38,5 & 48,8 & 45,7 & 42,9 \\
\hline \multicolumn{3}{|l|}{ Edad } & 38,5 & 37,5 & 36,2 & 41,2 \\
\hline \multirow{5}{*}{$\begin{array}{l}\text { País de } \\
\text { nacimiento }\end{array}$} & Territorios de lengua catalana & \multirow{5}{*}{ Sí } & 100 & 90,2 & 34,3 & 7,1 \\
\hline & Resto del Estado español & & 0 & 4,9 & 27,1 & 14,3 \\
\hline & Europa & & 0 & 1,2 & 24,3 & 71,4 \\
\hline & Suramérica & & 0 & 3,7 & 14,3 & 0 \\
\hline & Otros & & & & & 7,1 \\
\hline \multirow{4}{*}{$\begin{array}{l}\text { Cuantos años } \\
\text { hace que trabaja } \\
\text { en el PCB }\end{array}$} & Menos de 1 año & \multirow{4}{*}{ Sí } & 23,1 & 16,3 & 22,9 & 7,1 \\
\hline & Entre 1 y 3 años & & 38,5 & 26,3 & 37,1 & 71,4 \\
\hline & Entre 3 y 6 años & & 15,4 & 41,3 & 37,1 & 21,4 \\
\hline & Más de 6 años & & 23,1 & 16,3 & 2,9 & 0 \\
\hline \multirow{6}{*}{$\begin{array}{l}\text { Nivel de } \\
\text { estudios }\end{array}$} & Secundaria & \multirow{6}{*}{ Sí } & 0 & 0 & 0 & 7,1 \\
\hline & FP, ciclos formativos & & 16,7 & 1,3 & 1,4 & 0 \\
\hline & Licenciatura universitaria & & 8,3 & 29,5 & 23,2 & 0 \\
\hline & Máster & & 41,7 & 24,4 & 29 & 21,4 \\
\hline & Doctorado & & 25 & 21,8 & 17,4 & 35,7 \\
\hline & Estudios posdoctorales & & 8,3 & 23,1 & 29 & 35,7 \\
\hline \multirow{3}{*}{$\begin{array}{l}\text { Lengua de } \\
\text { respuesta }\end{array}$} & Catalán & \multirow{3}{*}{ Sí } & 100 & 86,6 & 12,9 & 14,3 \\
\hline & Castellano & & 0 & 11 & 74,3 & 7,1 \\
\hline & Inglés & & 0 & 2,4 & 12,9 & 78,6 \\
\hline \multirow{4}{*}{ L1 } & Solo o sobre todo en catalán & \multirow{4}{*}{ Sí } & 53,8 & 62,2 & 10 & 7,1 \\
\hline & $\begin{array}{l}\text { Igual en castellano que en } \\
\text { catalán }\end{array}$ & & 7,7 & 7,3 & 0 & 0 \\
\hline & Solo o sobre todo en castellano & & 38,5 & 28 & 60 & 14,3 \\
\hline & Otras lenguas o combinaciones & & 0 & 2,4 & 30 & 78,6 \\
\hline \multicolumn{3}{|c|}{ Media de conocimiento de catalán (saber hablar) } & 9,5 & 9,8 & 4,9 & 2,4 \\
\hline \multicolumn{3}{|c|}{ Media de conocimiento de castellano (saber hablar) } & 9,6 & 9,8 & 9,3 & 5,4 \\
\hline \multicolumn{3}{|c|}{ Media de conocimiento de inglés (saber hablar) } & 6,0 & 7,3 & 7,4 & 9,2 \\
\hline
\end{tabular}




\subsection{Grupo 1: investigadores autóctonos sin dominio del inglés}

El primero de los conglomerados está formado por investigadores que declaran tener un conocimiento alto de catalán hablado $(9,5$ de media) y castellano $(9,6)$, pero que, sin embargo, tienen un dominio intermedio del inglés (6) (Tabla 3). Todos los miembros de este grupo han nacido en algún territorio de habla catalana, y la mayoría de ellos $(53,8 \%)$ tiene el catalán como primera lengua (L1), mientras que un $38,5 \%$ declara el castellano como $\mathrm{L} 1$ y un $7,7 \%$ se declara bilingüe inicial catalán-castellano. Son predominantemente hombres $(61,5 \%)$ y tienen una media de edad de 38,5 años. En este sentido, de media no son apreciablemente mayores que el resto de investigadores analizados. Su vinculación al PCB sugiere que este colectivo parece incluir a dos subconjuntos: por un lado, más de la mitad lleva menos de 3 años en el PCB, mientras que casi una cuarta parte del colectivo lleva más de 6 años. Formativamente, también parecen percibirse dos colectivos: por un lado, casi el $17 \%$ está constituido por personal con formación profesional, lo que sugiere que se trata de técnicos de laboratorio. En el otro extremo destaca el porcentaje de personas con titulación de máster y doctorado, y el escaso porcentaje de estudios posdoctorales, lo que sugiere una estructura formativa más clásica que en el resto de colectivos.

Desde el punto de vista de los usos lingüísticos, este grupo está claramente instalado en el uso del catalán como lengua de trabajo prioritaria, con el castellano en una segunda posición y con el inglés poco presente (Tabla 4). Se trata de un grupo que no parece acudir masivamente a congresos, y en este ámbito muestra un comportamiento lingüístico que posterga el inglés. De hecho, el inglés solo aparece de manera remarcable en relación con las publicaciones de su ámbito profesional, ya sea en la lectura de revistas, de libros o de artículos de su especialidad, lo que hace suponer que se trata esencialmente de un uso receptivo. Cabe señalar que, de todos los colectivos analizados, es precisamente este el que utiliza más el castellano en las publicaciones especializadas, lo que parece denotar una función supletoria de esta lengua en el campo de la producción escrita científica, o quizá una especialización en la que esta lengua tenga algo más de presencia como idioma de publicaciones científicas. En cualquier caso, incluso en este grupo menos anglicizado, el inglés es la lengua más usada para estos menesteres. 
Tabla 4. Usos lingüísticos de los investigadores del grupo 1 ( $n=13)$

\begin{tabular}{|c|c|c|c|c|c|c|}
\hline & Indicadores & Catalán & Castellano & Inglés & $\begin{array}{l}\text { Otras } \\
\text { lenguas }\end{array}$ & Total \\
\hline \multirow{5}{*}{ 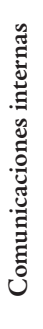 } & Hablar con sus compañeros de trabajo & 72,9 & 25,4 & 1,5 & 0,2 & 100 \\
\hline & $\begin{array}{l}\text { Hablar con sus jefes (director, investigador } \\
\text { principal) }\end{array}$ & 89,2 & 10,8 & 0,0 & 0,0 & 100 \\
\hline & $\begin{array}{l}\text { Hablar con el personal de gestión y } \\
\text { administración }\end{array}$ & 85,8 & 14,2 & 0,0 & 0,0 & 100 \\
\hline & $\begin{array}{l}\text { Escribir notas informales a sus compañeros de } \\
\text { trabajo (en papel, por correo electrónico, etc.) }\end{array}$ & 82,3 & 16,2 & 1,5 & 0,0 & 100 \\
\hline & Escribir documentos de gestión & 77,9 & 16,4 & 5,6 & 0,0 & 100 \\
\hline \multirow{7}{*}{ 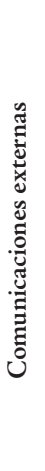 } & $\begin{array}{l}\text { Presentar comunicaciones en congresos de } \\
\text { ámbito internacional }\end{array}$ & 0,0 & 100,0 & 0,0 & 0,0 & 100 \\
\hline & $\begin{array}{l}\text { Presentar comunicaciones en congresos de } \\
\text { ámbito español o latinoamericano }\end{array}$ & 50,0 & 0,0 & 0,0 & 50,0 & 100 \\
\hline & $\begin{array}{l}\text { Presentar comunicaciones en congresos de } \\
\text { ámbito catalán }\end{array}$ & 0,0 & 0,0 & 0,0 & 0,0 & 0,0 \\
\hline & Hablar con investigadores de fuera de su grupo & 50,0 & 39,4 & 3,9 & 6,7 & 100 \\
\hline & Escribir artículos o trabajos de investigación & 16,8 & 42,8 & 40,3 & 0,0 & 100 \\
\hline & Leer libros relacionados con la disciplina & 0,9 & 33,6 & 65,5 & 0,0 & 100 \\
\hline & Leer revistas relacionadas con la disciplina & 0,5 & 24,1 & 75,5 & 0,0 & 100 \\
\hline
\end{tabular}

\subsection{Grupo 2: investigadores trilingües}

El grupo con mayor porcentaje de integrantes $(45,8 \%, \mathrm{n}=82)$ es el de los investigadores de L1 catalana $(62,2 \%)$ o L1 castellana $(28 \%)$ que declaran un nivel muy alto tanto de catalán como de castellano ( 9,8 en ambos casos) y alto de inglés $(7,3)$ (Tabla 3). Con una distribución muy homogénea entre hombres y mujeres ( $51,2 \%$ y $48,8 \%$ respectivamente) y una edad media de 37,5 años, se trata de un colectivo eminentemente autóctono o muy integrado, ya que un $90,2 \%$ ha nacido en los territorios de habla catalana, y casi todos los restantes son nacidos en el resto del Estado español o en Hispanoamérica. Desde una perspectiva profesional, la mayoría de sus integrantes lleva entre 3 y 6 años de vinculación con el PCB. Este es el colectivo con un nivel de formación medio más bajo, ya que la suma de licenciados y titulaciones inferiores apenas sobrepasa el 30\%.

Los investigadores de este conglomerado utilizan habitualmente el catalán, el castellano y el inglés, pero no lo hacen de forma aleatoria (Tabla 5), ya que utilizan básicamente dos lenguas para sus comunicaciones profesionales. Por un lado, se sirven principalmente del catalán tanto para relaciones orales como para usos escritos, internos o externos del PCB, incluyendo los congresos de ámbito 
catalán. Por otro lado, se sirven principalmente del inglés para las publicaciones académicas y los congresos internacionales. El castellano, por su parte, aparece como una lengua auxiliar, de uso apreciable en casi todos los casos, pero solo mayoritario en un ámbito muy concreto: los congresos de ámbito español o hispanoamericano.

Tabla 5. Usos lingüísticos de los investigadores del grupo $2(\mathrm{n}=84)$

\begin{tabular}{|c|c|c|c|c|c|c|}
\hline & Indicadores & Catalán & Castellano & Inglés & $\begin{array}{c}\text { Otras } \\
\text { lenguas }\end{array}$ & Total \\
\hline \multirow{5}{*}{ 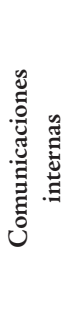 } & Hablar con sus compañeros de trabajo & 60,2 & 28,8 & 10,3 & 0,7 & 100 \\
\hline & $\begin{array}{l}\text { Hablar con sus jefes (director, investigador } \\
\text { principal) }\end{array}$ & 83,8 & 14,2 & 2,0 & 0,0 & 100 \\
\hline & $\begin{array}{l}\text { Hablar con el personal de gestión y } \\
\text { administración }\end{array}$ & 84,3 & 15,7 & 0,1 & 0,0 & 100 \\
\hline & $\begin{array}{l}\text { Escribir notas informales a sus compañeros de } \\
\text { trabajo (en papel, por correo electrónico, etc.) }\end{array}$ & 66,9 & 20,1 & 12,9 & 0,1 & 100 \\
\hline & Escribir documentos de gestión & 61,9 & 21,3 & 16,6 & 0,2 & 100 \\
\hline \multirow{7}{*}{ 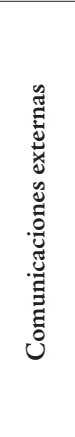 } & $\begin{array}{l}\text { Presentar comunicaciones en congresos de } \\
\text { ámbito internacional }\end{array}$ & 0,5 & 3,0 & 96,3 & 0,2 & 100 \\
\hline & $\begin{array}{l}\text { Presentar comunicaciones en congresos de } \\
\text { ámbito español o latinoamericano }\end{array}$ & 0,6 & 59,0 & 40,4 & 0,0 & 100 \\
\hline & $\begin{array}{l}\text { Presentar comunicaciones en congresos de } \\
\text { ámbito catalán }\end{array}$ & 70,2 & 3,5 & 26,3 & 0,0 & 100 \\
\hline & Hablar con investigadores de fuera de su grupo & 37,7 & 30,1 & 31,1 & 1,1 & 100 \\
\hline & Escribir artículos o trabajos de investigación & 5,6 & 4,8 & 89,6 & 0,0 & 100 \\
\hline & Leer libros relacionados con la disciplina & 1,0 & 4,8 & 93,9 & 0,3 & 100 \\
\hline & Leer revistas relacionadas con la disciplina & 0,7 & 2,4 & 96,6 & 0,2 & 100 \\
\hline
\end{tabular}

Los datos de las entrevistas con los investigadores permiten ilustrar la manera como los integrantes de este grupo estructuran sus usos lingüísticos. Por ejemplo, Pere (pseudónimo) trabaja habitualmente en tres lenguas (catalán, castellano e inglés) con una distribución basada no en ámbitos hipotéticos, sino sobre todo en quienes son sus interlocutores: catalán con catalanes o catalanohablantes, castellano con castellanohablantes e inglés con el resto de interlocutores:

ENTREVISTADOR: ¿Qué lenguas usas en el trabajo a lo largo de tu actividad?

Pere: Básicamente, tres. La gran mayoría de interacción con los compañeros de la oficina es en catalán, porque son catalanes. Ahora bien, yo en particular, trabajo con dos personas más dentro de mi equipo una de las cuales es [de nacionalidad hispanoamericana] y la otra es [de nacionalidad europea] y, aunque están aprendiendo catalán, acabamos hablando en castellano con ellos dos la mayoría del tiempo, aunque, ya te digo, se 
intenta pero xxx en la práctica el resultado es este: catalán con la mayoría de compañeros, castellano con estos dos en particular, hay uno de [origen español] pero a este le intentamos machacar más, y después inglés. (Traducción de los autores).

La cita de Pere — que domina el inglés después de haber estudiado y trabajado bastantes años en un país anglófono- muestra que el inglés no es necesariamente la lengua franca con los alóglotas: de hecho, en el PCB, como en el resto de la sociedad catalana, el castellano es la lengua que funciona a menudo de interrelación con los alóglotas, incluso como puente antes de que aprendan catalán. La cita muestra también como el esbozo de distribución funcional que presentan los datos generales tiene que matizarse en función de cada unidad de investigación concreta, ya que estas son verdaderas comunidades de prácticas en las que se generan dinámicas autónomas. Teniendo en cuenta la composición demolingüística del PCB y de este equipo concreto, se puede afirmar que el catalán es la lengua predominante en las relaciones internas, con el resto del PCB y con la sociedad catalana en general, tanto de manera oral como escrita. De hecho, Pere declara que «un $90 \%$ del tiempo» habla en catalán con los trabajadores del PCB.

\subsection{Grupo 3ः investigadores bilingües en castellano e inglés}

El tercer conglomerado es el formado por 70 investigadores $(39,1 \%$ del total), cuya L1 es el castellano u otra lengua que no sea el catalán $(60 \%$ y $30 \%$, respectivamente) (Tabla 3). Es un colectivo que domina el castellano, puede usar el inglés con fluidez y tiene habilidades receptivas, pero no productivas, en catalán. Se trata de un colectivo integrado por investigadores de procedencia más diversa que los dos grupos anteriores: los nacidos en los territorios de lengua catalana son un $34,4 \%$, los procedentes del resto del Estado español son un 27,1\% y el resto procede de Europa (24,3\%) o Hispanoamérica (14,3\%). En términos socioeconómicos guarda bastantes semejanzas con el colectivo anterior, aunque es ligeramente más joven, tiene una vinculación laboral algo menor en términos de años de trabajo en el PCB y tiene un nivel de formación algo más elevado.

El comportamiento de este tercer grupo reproduce en cierta medida los patrones identificados para el grupo de los autóctonos con dominio del inglés (Tabla 6). Por un lado, tal como muestra la Tabla 6, este grupo se sirve principalmente del inglés en publicaciones de su ámbito. Ahora bien, este colectivo utiliza el castellano como lengua habitual en sus actividades laborales cotidianas y hace un uso del catalán más bien escaso, utilizándolo en porcentajes apreciables en relaciones de ámbito catalán, en las relaciones con el personal de administración y en los congresos catalanes. 
Tabla 6. Usos lingüísticos de los investigadores del grupo $3(\mathrm{n}=70)$

\begin{tabular}{|c|c|c|c|c|c|c|}
\hline & Indicadores & Catalán & Castellano & Inglés & $\begin{array}{c}\text { Otras } \\
\text { lenguas }\end{array}$ & Total \\
\hline \multirow{5}{*}{ 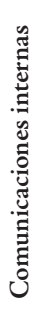 } & Hablar con sus compañeros de trabajo & 12,4 & 72,5 & 13,5 & 1,5 & 100 \\
\hline & $\begin{array}{l}\text { Hablar con sus jefes (director, investigador } \\
\text { principal) }\end{array}$ & 14,1 & 75,0 & 10,9 & 0,0 & 100 \\
\hline & $\begin{array}{l}\text { Hablar con el personal de gestión y } \\
\text { administración }\end{array}$ & 19,6 & 78,7 & 1,8 & 0,0 & 100 \\
\hline & $\begin{array}{l}\text { Escribir notas informales a sus compañeros de } \\
\text { trabajo (en papel, por correo electrónico, etc.) }\end{array}$ & 8,5 & 77,4 & 13,3 & 0,7 & 100 \\
\hline & Escribir documentos de gestión & 17,3 & 65,2 & 17,4 & 0,0 & 100 \\
\hline \multirow{7}{*}{ 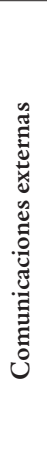 } & $\begin{array}{l}\text { Presentar comunicaciones en congresos de } \\
\text { ámbito internacional }\end{array}$ & 0,1 & 6,4 & 93,4 & 0,2 & 100 \\
\hline & $\begin{array}{l}\text { Presentar comunicaciones en congresos de } \\
\text { ámbito español o latinoamericano }\end{array}$ & 0,0 & 58,3 & 41,7 & 0,0 & 100 \\
\hline & $\begin{array}{l}\text { Presentar comunicaciones en congresos de } \\
\text { ámbito catalán }\end{array}$ & 45,9 & 30,2 & 23,9 & 0,0 & 100 \\
\hline & Hablar con investigadores de fuera de su grupo & 8,6 & 52,2 & 37,7 & 1,5 & 100 \\
\hline & Escribir artículos o trabajos de investigación & 0,9 & 13,9 & 85,2 & 0,0 & 100 \\
\hline & Leer libros relacionados con la disciplina & 0,8 & 12,1 & 86,3 & 0,8 & 100 \\
\hline & Leer revistas relacionadas con la disciplina & 0,5 & 6,7 & 92,5 & 0,3 & 100 \\
\hline
\end{tabular}

El caso de Alberto (pseudónimo), un investigador hispanoamericano formado en un país anglófono y llegado a Cataluña un año antes de realizar la entrevista, ejemplifica el perfil del investigador de este grupo: domina el castellano y el inglés pero tiene escasos conocimientos de catalán y, a pesar de su voluntad, expresada varias veces, de aprender catalán, sus usos cotidianos pivotan en torno al castellano o el inglés. De hecho, se muestra muy favorable a potenciar el uso y el aprendizaje del inglés entre sus colegas. En realidad, Alberto se había formado técnicamente en inglés y declara que a menudo recurre a este idioma para la terminología específica, aunque menos que en los inicios de su incorporación al PCB:

Entrevistador: ¿Cuáles son las lenguas que utilizas habitualmente?

Alberto: Castellano e inglés, y cuando no tengo control del asunto, pues catalán.

Entrevistador: ¿Cuando no tienes control del asunto?

Alberto: Cuando no puedo, porque, o sea, sí, cuando, en el laboratorio hay gente de habla inglesa, y de catalana y castellana, entonces los de habla inglesa generalmente no dominan muy bien el castellano y aprovecho yo 
para, el inglés es un idioma importante en el trabajo nuestro, entonces eso está bien hablar en inglés cuando se puede, y cuando no, cuando solo xxx personas, hablamos en castellano. A mí me gusta que se hable en catalán para yo tratar de aprender pero generalmente ya la gente empieza [en castellano].

\subsection{Grupo 4: investigadores instalados en el inglés}

El último grupo se compone de investigadores que dicen utilizar básicamente el inglés para sus quehaceres profesionales. Este comportamiento guarda relación con sus competencias lingüísticas: su nivel de inglés $(9,2)$ es claramente superior a su nivel intermedio de castellano $(5,4)$ y de catalán $(2,4)$ (Tabla 3). Se trata de un colectivo nacido fuera de España e Hispanoamérica, con ligero predominio masculino, una vinculación laboral reciente con el PCB y un acusado predominio de personas con estudios doctorales y posdoctorales. Es el grupo que tiene una media de edad superior (41 años). Cabe señalar, sin embargo, que incluye a un porcentaje considerable (21\%) de investigadores nacidos en los territorios de lengua catalana o en el resto del Estado español, que presumiblemente deben encontrarse en equipos de trabajos con fuerte componente internacional. La presencia de investigadores catalanohablantes o castellanohablantes en este colectivo eleva las medias de conocimiento de catalán y castellano de todo el grupo, que sin estos integrantes serían aún más bajas.

Tal como muestra la Tabla 7, el inglés es la lengua dominante de este colectivo para todas las funciones y ámbitos analizados, con porcentajes superiores al $71 \%$ de los usos. La única excepción es el uso del castellano y el catalán con el personal de administración y gestión del PCB.

Tabla 7. Usos lingüísticos de los investigadores del grupo 4 (n=14)

\begin{tabular}{|c|c|c|c|c|c|c|}
\hline & Indicadores & Catalán & Castellano & Inglés & $\begin{array}{c}\text { Otras } \\
\text { lenguas }\end{array}$ & Total \\
\hline \multirow{5}{*}{ 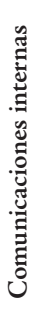 } & Hablar con sus compañeros de trabajo & 5,4 & 18,9 & 71,1 & 4,6 & 100 \\
\hline & $\begin{array}{l}\text { Hablar con sus jefes (director, investigador } \\
\text { principal) }\end{array}$ & 12,5 & 13,6 & 73,9 & , & 100 \\
\hline & $\begin{array}{l}\text { Hablar con el personal de gestión y } \\
\text { administración }\end{array}$ & 17,1 & 53,6 & 29,3 & , 0 & 100 \\
\hline & $\begin{array}{l}\text { Escribir notas informales a sus compañeros de } \\
\text { trabajo (en papel, por correo electrónico, etc.) }\end{array}$ & , 0 & 5,0 & 92,9 & 2,1 & 100 \\
\hline & Escribir documentos de gestión & 7,9 & 9,3 & 82,9 & 0 & 100 \\
\hline
\end{tabular}




\begin{tabular}{|c|l|c|c|c|c|c|}
\hline & Indicadores & Catalán & Castellano & Inglés & $\begin{array}{c}\text { Otras } \\
\text { lenguas }\end{array}$ & Total \\
\hline \multirow{2}{*}{$\begin{array}{l}\text { Presentar comunicaciones en congresos de } \\
\text { ámbito internacional }\end{array}$} &, 0 &, 0 & 100,0 &, 0 & 100 \\
\cline { 2 - 6 } & $\begin{array}{l}\text { Presentar comunicaciones en congresos de } \\
\text { ámbito español o latinoamericano }\end{array}$ &, 0 & 1,4 & 98,6 &, 0 & 100 \\
\cline { 2 - 7 } & $\begin{array}{l}\text { Presentar comunicaciones en congresos de } \\
\text { ámbito catalán }\end{array}$ &, 0 & 1,4 & 98,6 &, 0 & 100 \\
\cline { 2 - 7 } & Hablar con investigadores de fuera de su grupo & 1,4 & 11,8 & 80,7 & 6,1 & 100 \\
\cline { 2 - 7 } & Escribir artículos o trabajos de investigación &, 0 &, 0 & 100,0 &, 0 & 100 \\
\cline { 2 - 7 } & Leer libros relacionados con la disciplina &, 0 &, 0 & 96,2 & 3,8 & 100 \\
\cline { 2 - 7 } & Leer revistas relacionadas con la disciplina &, 0 &, 0 & 99,6 &, 4 & 100 \\
\hline
\end{tabular}

\section{Conclusiones}

El análisis del personal investigador del PCB permite entrever cuatro grandes grupos lingüísticos en función del origen, las competencias lingüísticas y los comportamientos idiomáticos que exhiben en sus actividades profesionales.

El primero de los grupos es porcentualmente reducido y está formado por investigadores autóctonos que dominan el catalán y el castellano pero solo tienen habilidades intermedias en inglés. Es un colectivo asentado en el uso del catalán y que tiene el castellano como segunda lengua de interacción. El segundo grupo, el mayor en términos de integrantes, está formado básicamente por investigadores autóctonos, capaces de utilizar el catalán, el castellano y el inglés. Estos investigadores se sirven de las tres lenguas con cierta especialización funcional: el catalán para usos internos y de relación con su entorno social, el castellano para las relaciones con interlocutores hispanófonos y el inglés para relaciones exteriores $y$, sobre todo, para la publicación y el consumo de materiales científicos. El tercer colectivo está formado por investigadores procedentes mayormente de territorios hispanófonos, capaces de usar el castellano y el inglés, pero no el catalán. Distribuyen estos dos idiomas de manera parecida al anterior, con el castellano asumiendo las funciones de lengua para las relaciones de proximidad. Finalmente, el cuarto colectivo está formado principalmente por investigadores procedentes de fuera de las áreas lingüísticas catalana e hispana, y se caracterizan por servirse del inglés como lengua de comunicación habitual en sus relaciones profesionales. Este último grupo incluye a algunos investigadores locales, presumiblemente integrados en equipos de trabajo con numerosos extranjeros.

$\mathrm{El}$ análisis de los comportamientos de cada uno de los cuatro grupos permite vislumbrar una serie de regularidades en las prácticas comunicativas del personal investigador del PCB. En este sentido, se percibe una clara diferencia en- 
tre las actividades que podemos denominar de consumo y proyección científica - lectura de bibliografía especializada, producción de artículos especializados, participación en congresos internacionales - y las actividades profesionales cotidianas, vinculadas a la gestión y las relaciones interpersonales. Las primeras están claramente decantadas hacia el inglés en todos los grupos, incluso en aquel integrado por autóctonos que no dominan esta lengua. La única excepción a esta inclinación es la lengua elegida en los congresos hispanoamericanos y españoles, y en los congresos catalanes, donde el entorno y los participantes invitan a un uso mayoritario del castellano y el catalán, respectivamente. Cabe señalar, sin embargo, que el uso del inglés en este tipo de eventos es también considerable. Las actividades profesionales cotidianas, por su parte, se desarrollan mayoritariamente en catalán, lengua usada por el primer y el segundo grupo. Los investigadores que no conocen la lengua local pero tienen conocimientos de castellano tienden a usar esta lengua como lengua de comunicación con sus colegas, mientras que en estos menesteres el inglés queda reservado sobre todo para aquellos que no saben ni catalán ni castellano.

Esta investigación confirma que los procesos de cambio lingüístico no se producen de manera compacta sino sectorial, de acuerdo con equilibrios complejos que dependen de la constitución y los equilibrios de cada ecosistema lingüístico. En este caso particular, el proceso de internacionalización inherente a la investigación científica no ha comportado la desaparición de las lenguas distintas del inglés. Sin duda alguna, el inglés se ha convertido en la lengua de consumo y proyección de la ciencia, y todos los miembros de la institución investigada, incluso los menos proclives a ello, han adquirido cierto nivel en esta lengua. Sin embargo, el haber adquirido este estatus no ha convertido la lengua de Newton y Darwin en la lengua por defecto de la institución estudiada. De hecho, se percibe una jerarquía por la que la primera lengua usada en la mayoría de funciones es el catalán, seguido del castellano, y el inglés solo en tercer lugar; una jerarquía lógicamente aparejada a las competencias de los hablantes y a la demolingüística de su población, pero también dependiente de la gestión lingüística de la institución. En otras palabras, la respuesta a la pregunta con la que encabezábamos este artículo — ¿en qué lengua se hace ciencia? - tiene, en el caso del PCB, una respuesta plural: en varias lenguas, básicamente el catalán, el castellano y, cómo no, también el inglés.

Así pues, los resultados el estudio del PCB parecen desmentir las concepciones más simplistas de la globalización como proceso de unificación lingüística en todos los planos. Ciertamente, el inglés se ha establecido como lengua franca científica - o sea, usada para la intercomunicación en caso de diversidad lingüís- 
tica- $y$, en este sentido, en cierto modo excluye a otros idiomas competidores potenciales como lengua de proyección científica. Es muy posible que en contextos poscoloniales y en entornos de composición demolingüística muy diversificados, el inglés funcione efectivamente como lengua de trabajo. Pero en ecosistemas donde coincide con otras lenguas completas (Lamuela, 1994) —incluso allá donde hay más de una, como en el caso analizado aquí-, no está en absoluto claro que asuma todas las funciones comunicativas. El estudio ha detectado un pequeño porcentaje de investigadores autóctonos que declara usar el inglés como principal lengua de trabajo, pero también es cierto que se han detectado investigadores que, sin tenerlas como lenguas iniciales, han adoptado el castellano e incluso el catalán como lengua laboral en el PCB. En este sentido, conviene recordar que el catalán no solo es la lengua propia de Cataluña y oficial, sino que es la lengua de funcionamiento por defecto de la administración del PCB, por lo que su aprendizaje resulta de utilidad. La introducción de cambios en este sentido implicaría una reducción de su utilidad en la comunidad científica aquí analizada. 


\section{Bibliografía}

Ammon, Ulrich (1999). «Deutsch als Wissenschaftsprache: die Entwincklung im 20 Jahrhundert, und die Zukunftperspektive», en Herbert Ernst Wiegend (ed.) (1999). Sprache und Sprachen in den Wissenschaften: Geschichte und Gegenwart. Berlin, Nueva York: De Gruyter.

Аммом, Ulrich (2010). «World languages: Trends and futures», en Nikolas Coupland (ed.) (2010). The handbook of language and globalization. Chichester: Wiley-Blackwell.

Aracil, Lluís Vicent (2004). Do Latim às Linguas Nacionais: Introdução à Historia Social das Linguas Europeias. Santiago de Compostela: Associação de Amizade Galiza-Portugal.

Arzoz, Xabier (2012). Bilingual education in the legal context. Group rights, State policies and globalisation. Leiden, Boston: Martinus Neijhoff.

Baggioni, Daniel (1997). Langues et nations en Europe. París: Éditions Payot \& Rivages.

Bastardas i Boada, Albert (2007). Les polítiques de la llengua i la identitat a l'era «glocal». Barcelona: Generalitat de Catalunya, Departament d'Interior, Relacions Institucionals i Participació, Institut d'Estudis Autonòmics.

Boix i Fuster, Emili y F. Xavier Vila i Moreno (1998). Sociolingüistica de la llengua catalana. Barcelona: Ariel.

Bourdieu, Pierre (1982). Ce que parler veut dire. L'économie des échanges linguistiques. Poitiers: Fayard. Trad. al castellano: (1985) ¿Qué significa hablar? Economía de los intercambios lingüísticos. Torrejón de Ardoz: Akal.

Calvet, Louis-Jean (1999). Pour une écologie des langues du monde. París: Plon.

- y Lía Varela (2000). «xxie siècle: le crépuscle des langues? Critique du discours politico-linguistiquement correct». Estudios de Sociolingüistica, 1: 47-64.

Coupland, Nikolas (2010). «Introduction: Sociolinguistics in the global era», en Nikolas Coupland (ed.) (2010). The handbook of language and globalization. Chichester Wiley-Blackwell.

De Swann, Abram (2001). Words of the world. The global language system. Malden: Polity Press.

- (2010). «Language systems», en Nikolas Coupland (ed.) (2010). The handbook of language and globalization. Chichester: Wiley-Blackwell.

Eckert, Penelope y Sally McConnell-Ginet (1999). «The Community of Practice: Theories and methodologies in language and gender research». Language in Society, 28: 185-201.

FAirClough, Norman (2006). Language and globalization. Londres: Routledge. 
Fishman, Joshua (1999). «The new linguistic order». Foreign Policy, 113: 26-40. GaL, Susan (1987). «Linguistic repertoire», en Ulrich Ammon, Norbert Dittmar y Klaus Mattheier (1987). Sociolinguistics - Soziolinguistik, Vol. I. Berlin: DeGruyter.

Graddol, David (2006). English next. Why Global English may mean the end of 'English as a Foreign Language. <http://www.britishcouncil.org/learningresearch-english-next.pdf>.

Held, David; Anthony McGrew; David Goldblatt y Jonathan Perraton (1999). Global transformations: Politics, economics and culture. Cambridge: Polity.

Heller, Monica (2002). Éléments d'une sociolinguistique critique. París: Didier. Kachru, Braj; Yamuna Kachru y Cecil Nelson (2006). The handbook of World Englishes. Chichester: Wiley.

Mufwene, Salikoko S. (2008). Language evolution. Contact, competition and change. Londres, Nueva York: Continuum.

Mufwene, Salikoko S. (2010). «Globalization, global English, and World English(es): Myths and facts», en Nikolas Coupland (ed.) (2010). The handbook of language and globalization. Chichester: Wiley-Blackwell.

Nettle, David y Suzanne Romaine (2000). Vanishing voices: The extinction of the world's languages. Oxford: Oxford University Press.

Ostrer, Nicholas (2010). The last lingua franca. English until the return of Babel. Londres, Nueva York: Allen Lane.

Phillipson, Robert (1992). Linguistic imperialism. Oxford: Oxford University Press.

Phillipson, Robert (2003). English-only Europe? Challenging language policy. Londres: Routledge.

Phillipson, Robert (2006). «Language policy and linguistic imperialism», en Thomas Ricento (ed.) (2006). An introduction to language policy. Theory and method. Oxford: Blackwell.

Pool, Thomas (2010). «Panlingual globalization», en Nikolas Coupland (ed.) (2010) The handbook of language and globalization. Chichester: WileyBlackwell.

Renau, E. (dir.).(2004). «Enquesta d'usos lingüístics a les institucions públiques catalanes». Ejemplar fotocopiado.

Ricento, Thomas (2010). «Language policy and globalization», en Nikolas Coupland (ed.) (2010). The handbook of language and globalization. Chichester: Wiley-Blackwell. 
Romaní, Josep M. (2005). «La variabilitat en els usos lingüístics a les empreses catalanes: factors i tendències», en Generalitat de Catalunya (2005). Els usos lingüistics a les empreses amb vocació internacional. Barcelona: Secretaria de política lingüística.

Skutnabb-Kangas, Tove y Robert Phillipson (2010). «The global politics of language: Markets, maintenance, marginalization, or murder?», en Nikolas Coupland (ed.) (2010). The handbook of language and globalization. Chichester: Wiley-Blackwell.

Vila i Moreno, F. Xavier (2012). «Algunes bases per a la recerca sociolingüística en sentit ampli» en F. Xavier Vila i Moreno (dir.) (20120). Posar-hi la base. Usos $i$ aprenentatges lingüístics en el domini català. Barcelona: Institut d'Estudis Catalans. Acesible en: <http://publicacions.iec.cat/Front/PopulaFitxa.do?moduleName $=$ novetats_editorials $\&$ subModuleName $=$ darrer es_novetats\&idCatalogacio $=17208>$.

Vila i Moreno, F. Xavier (2012). Survival and development of language communities. Prospects and challenges. Clevedon: Multilingual Matters.

Vila i Moreno, F. Xavier y Vanessa Bretxa (en preparación). Lingua Academica. Language policy in bigher education.

Vila i Moreno, F. Xavier; Vanessa Bretxa y Llorenç Comajoan (2012). «Llengües i globalització en el món de la recerca: els coneixements i els usos científics al Parc Científic de Barcelona». Caplletra, 52:35-64.

Vila i Moreno, F. Xavier; Llorenç Comajoan, Vanessa Bretxa y Liliana Arroyo (2009). «El multilingüisme al Parc Científic de Barcelona: Coneixements, usos i necessitats lingüístics». Centre Universitari de Sociolingüística i Comunicació CUSC-PCB/UB, Barcelona. 